that with $r_{\mathrm{i}}=1,500 \mathrm{~km}$, the IG-soliton GRS drift speed would be $10 \mathrm{~m} \mathrm{~s}^{-1}$ which is outside $-3.5 \pm 5 \mathrm{~m} \mathrm{~s}^{-1}$.

Our experiments and simulations show that in a turbulent QG shear flow, vortices grow and are sustained by merging. Similarly, 24 mergers of vortex pairs in the same zone were observed by Voyager ${ }^{17}$. Antipov et al. also observed merging, but merging per se occurs in a wide variety of flows. The mergers Antipov et al. observed were not in a turbulent shear flow and not in the regime where they observed a persistent solitary vortex (refs 3,6-8 describe merging of transient vortices generated by a rotating disk in a laminar flow). The striking feature of the vortices on Jupiter and in our experiments is their persistence in the presence of turbulence. In a rapidly rotating tank, there is fast dissipation. The vortices of Antipov et al. survive longer than the Ekman friction time because the fluid layer is locally thicker at the vortex. In the surrounding thin fluid layer, however, the turbulence is strongly damped by Ekman friction. Thus, the vortices of Antipov et al. are laminar.

Although we disagree with Antipov et al.'s identification of the GRS as an IG soliton, we are fascinated by their experiments and would like to understand them better. Measurements of vortex size and shape as a function of fluid depth and shear strength would be helpful.

PHILIPS. MARCUS Department of Mechanical Engineering, University of California, Berkeley, California 94720, USA

Madylam, ENSHMG, JOEL SOMMERIA

BP 95, 38420 St Martin D'Heres,

France

SteVEn D. Meyers HARRY L. SWINNEY

Department of Physics,

University of Texas,

Austin, Texas 78712, USA

1. Sommeria, J., Meyers, S. D. \& Swinney, H. L. Nature 331. 689-693 (1988)

2. Marcus, P. S. Nature 331, $693-696$ (1988)

3. Nezlin, M. V. Soviet Phys. Usp. 29, 817-842 (1986)

4. Williams, G. P. \& Yamagata, T. J. atmos. Sci. 41, 453478 (1984).

5. Dowling, T. E. \& Ingersoll, A. P. J. atmos. Sci. 45, 1380 1396 (1988).

6. Antipov, S. V., Nezlin, M. V., Snezhkin, E. N. \& Trubnikov, A. S. Soviet Phys. JETP 62, 1097-1107 (1985).

7. Antipov, S. V., Nezlin, M. V., Snezhkin, E. N. \& Trubnikov, A. S. Nature 323, 238-240 (1986).

8. Antipov, S. V., Nezlin, M. V., Rodionov, V. K., Snezhkin, E. N. \& Trubnikov. A. S. Soviet Phys. JETP 57, 786-801 (1983).

9. Beebe, R. \& Hockey, T. A. Icarus 67, 96-105 (1986)

10. Maxworthy, T. \& Redekopp, L. G. Icarus 29, 261-271 (1976).

11. Antipov, S. V. et al. Fizika Plazmy 14, 1104-1121 (1988).

12. Pedlosky, J. Geophysical Hydrodynamics (Springer, New York, 1982).

13. Cederlöf, U. J. Fluid Mech. 187, 395-406 (1988).

14. Lindley, D. Nature 341, 95 (1989)

14. Lindley, D. Nature 341,95 (1989).
15. Marcus, P. S. \& Van Buskirk, R. D. J. atmos. Sci. (subMarcus,
mitted).

16. Dowling, T. E. \& Ingersoll, A. P. J. atmos. Sci. 45, 1380 1396 (1988)

17. Mac Low, M.-M \& Ingersoll, A. P. Icarus 65, 353-369 (1986).

18. Williams, G. P. \& Wilson, R. J. J. atmos. Sci. 45, 207241 (1988).

\title{
Ubihomologous homology usage
}

SIR-The body of scientific ideas often grows faster than the vocabulary available for its expression, but the imprecise use of the word 'homology' in molecular biochemistry to mean 'sequence similarity' is unnecessary and can be obstructive because the two concepts conveyed by the single word are related ${ }^{1}$.

The tendency in biology to assign homology to various generalizable associations manifests itself further in immunology, where the word 'homologous' takes on at least two additional meanings. In one sense, 'homologous' is used, largely in molecular immunology but also in other areas, to describe the relationship of an antibody with its naturally eliciting antigen, and stands in contrast to heterologous. By this definition, the combining site of anti-bovine serum albumin antibody is homologous with bovine serum albumin and heterologous with bovine myoglobin. Thus, 'homology' implies a causal physiological link, usually in association with a high degree of structural complementarity. (It does not refer to the structural complementarity itself.)

Unfortunately, this is not the only meaning of homology in immunology. 'Homologous', in its broad sense, also refers to the relationship of an antigen to an immunogenic host of the same species. Bovine serum albumin (BSA) is thus homologous to cows but heterologous to rabbits. Is rabbit anti-bovine serum albumin antibody, then, homologous or heterologous to BSA? Many immunologists would say the answer depends upon the context in which the question is asked, and that the intention would be readily understood by anyone working in the field.

The terminological ambiguity increases in more complicated cases. Suppose an immunologist is investigating two chemically distinct forms of bovine serum albumin, $\mathrm{BSA}_{1}$ and $\mathrm{BSA}_{2}$. He immunizes a rabbit with $\mathrm{BSA}_{1}$ and finds that some of the antibodies elicited react with $\mathrm{BSA}_{2}$. What are the relationships of $\mathrm{BSA}_{1}$ and $\mathrm{BSA}_{2}$ to these antibodies? Some molecular immunologists might say $\mathrm{BSA}_{1}$ is the homologous antigen whereas $\mathrm{BSA}_{2}$ is a heterologous antigen, even though $\mathrm{BSA}_{1}$ and $\mathrm{BSA}_{2}$ are from the same species and, in this more general biological sense, are both heterologous to the antibodies.

Alternatively, suppose a cow is immunized with rabbit serum albumin and the antibodies found to react with BSA. Such results have often been observed ${ }^{2}$. Is BSA in this instance a heterologous antigen? And what of the relation between rabbit anti-BSA antibody and the antibody it elicits in a cow? Would the eliciting and elicited antibodies in this case be referred to as heterologous? As antibodies produced by different species, they are. As an antibody-antigen pair, they are not.
The potential for confusion in the description of antibody-antigen reactions also arises in that of cellular receptor-antigen interactions.

Although immunologists generally recognize the terms as they apply in their own field, for the sake of consistency throughout immunology and biology, the terminology needs to be clarified.

We propose that the use of the words homologous and heterologous in biology be reserved for the indication of suggestion of common phylogenetic ancestry or the lack thereof. Relationships among and within species may be aptly described by such terms as allogeneic, xenogeneic and syngeneic as in xenogeneic graft, allogeneic antigen (or simply 'alloantigen'). As for the relationship between antibody and antigen or between receptor and antigen, we suggest the introduction of two new terms: homoligous (homo-LYE-gous) and heteroligous (hetero-LYE-gous), the former referring to an eliciting elicitedligand-receptor pair and the latter to a pair lacking the causal link and describable in terms of degree of crossreactivity.

ROBERT J. PETRELla

MiTCHEL M. YOKOYAMA

Department of Immunology

Kurume University School of Medicine

Kurume,

Japan

1. Reeck, G.R. et al. Cell50, 667 (1987)

2. Twining, S.S., Lehmann, H. \& Atassi, M.Z. Biochem. J. $191,681-697(1980)$.

\section{All shook up}

SIR-The recent experiments ${ }^{1}$ (see ref. 2), in which a spinning gyroscope apparently loses weight, have an obvious flaw: anyone who has seen components travelling on a vibration-feed conveyer will have doubts about weighing vibrating objects.

Gyroscopes contain spinning material and so must vibrate. Given that gyroscope bearings are imperfect, the vibration could consist of a linear movement followed by a return movement - at a slightly different speed. This asymmetric vibration will interact with the damping mechanisms in the weighing device. For example, mechanical friction would be less for the faster movement. So pivot bearings in a balance could exert a thrust on the gyroscope being weighed. The mystery is no more.

\section{Cromwell Road}

RICHARD BAKER

\section{Kingston KT2 6RE}

UK

1. Hayasaka, H. \& Takeuchi, S. Phys. Rev. Lett. 63, 2701-
2704 (1989).
2. Maddox, J. Nature 343, 113 (1990).

- See also S.H. Salter's fuller description of this effect on page 509 of this issue. 